\title{
GENTLE: Reducing Reader Collision in Mobile RFID Networks*
}

\author{
Jieun $\mathrm{Yu}$ and Wonjun $\mathrm{Lee}^{+}$ \\ Network Research Lab., Dept. of Computer Science and Engineering, \\ Korea University, Seoul, Republic of Korea \\ wlee@korea.ac.kr
}

\begin{abstract}
Mobile RFID, the technology of a cellular phone equipped with a RFID reader, allows users to read RFID tags anywhere. However, signals from more than two readers can interfere with one another, i.e., reader collision problem; reliable tag reading is necessary. This paper proposes Gentle protocol to use beacon messages and multi-channel for increasing throughput. In addition, Gentle protocol can put tag ID information into the beacon message and share it among close readers. Simulation results show that Gentle protocol outperforms existing reader anticollision protocols.
\end{abstract}

Keywords- Mobile RFID, Reader Anti-Collision

\section{Introduction}

One of emerging wireless technologies, Radio Frequency Identification (RFID) system which will soon replace the bar codes is increasingly being used in supply chain. The RFID system consists of Radio Frequency (RF) readers and tags. Usually readers send queries to their tags for the stored information. Tags that are normally attached onto objects transmit the object-related information to readers as requested. What is beneficial from using RFID system is that it allows a reader to read tags in long distance and also obtain information from several tags simultaneously. The RFID system, which has already been adopted in applications such as supply chain, is now considered a front-runner of a ubiquitous era with emergence of Mobile RFID [9][15]. In Mobile RFID, the reader is installed in a cellular phone and services are provided over a telecommunication network. For example, Mobile RFID enables clients to scan tags attached on movie posters and receive URL information for the movies so that clients can watch movie trailers or buy tickets.

However, there exist two collision problems that reduce read throughput (number of tags read per time unit). Tag Collision [10] occurs when multiple tags respond to a reader and the reader can not identify signals from the tags accordingly. Reader Collision [1] arises when multiple readers are in close proximity and therefore the readers interfere with one another and can not read tags. In Mobile RFID, reader collision is regarded a more severe problem than tag collision because of its distinctive characteristics that reading many tags at once is not necessary and readers usually move around. Possible reader collision's causes are 1) absence of functionality in tags that prevents the reader collision due to their cheap price and 2) that a reader's signal is much stronger than that of a tag's because most tags do not have a battery but instead obtain its operational power from the reader's signal. In practice, transmission ranges of a tag and a reader in Mobile RFID environment are up to 1 meter and several hundred meters respectively. In other words, a reader can read tags within 1 meter but the signal of the reader affects other readers within several hundred meters. Consequently, a customized reader anti-collision protocol to RFID network is required.

For the RFID reader collision problem, there have been various solutions proposed [1][2][6][11]. The previous solutions usually propose TDMA scheduling techniques [1][2] which consider static readers only. It means that they are not efficient when using mobile readers. Furthermore, in early studies, multi-channel is not assumed to be operational whereas in reality, RFID standard [3][4] exploits multi-channel to circumvent reader collision. However, multi-channel environment is also not enough to restrain all kinds of reader collision. There is only a single paper [6] that examines

\footnotetext{
* This work was jointly supported by a grant from SK Telecom, Korea [Project No. KU-R040572], by the Korea Science and Engineering Foundation(KOSEF) grant funded by MEST (No. R01-2007-000-11203-0), and by MKE, Korea under ITRC IITA-2008-(C1090-0801-0046).

+ Corresponding Author
} 


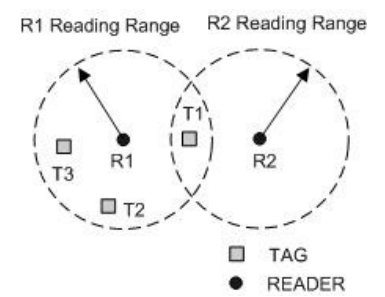

(a) Multiple reader-to-tag collision

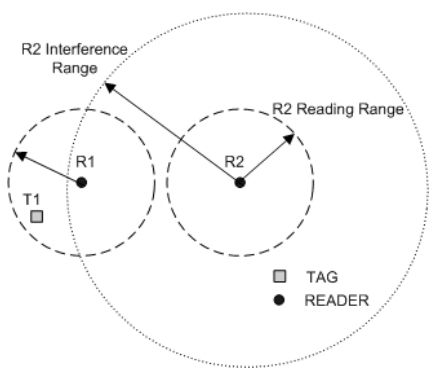

(b) Reader-to-reader collision

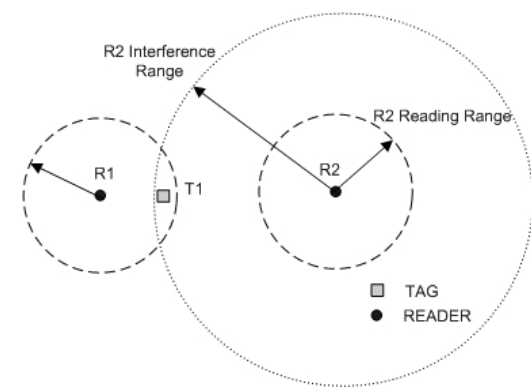

(c) Tag hidden terminal problem

Figure 1. Reader collision problems

reader collision with movable readers based on CSMA technique but it can not use multi-channel due to beacon messages.

This paper proposes a distributed CSMA based mechanism called Gentle that uses RFID multi-channel and beacon messages to mitigate reader collision. In addition, readers can put tag information in their beacon messages in order to forward the information to their close readers. By using the two methodologies, it is shown in Gentle that readers avoid reader collision more efficiently and reduce waiting time of getting tag information such that improves the previous CSMA based anti-reader collision mechanism [4][6].

The rest of the paper is organized as follows. We first present three types of collision problems in RFID systems in Section 2. We then present related work in Section 3, followed by the description of the main algorithm, RFID Gentle protocol in Section 4. Section 5 analyze performance of our algorithm with simulation results and in Section 6, we conclude our paper.

\section{RFID Reader Collision Problem}

There are three types of reader collision: multiple reader-to-tag collision, reader-to-reader collision, and tag hidden terminal problem. A reader can read tags only in its reading range and other nearby readers can detect the signal of the reader in its interference range.

\subsection{Multiple Reader-to-Tag Collision}

As illustrated in Figure 1 (a), when readers R1 and R2 transmit query messages to a tag $\mathrm{T} 1$, $\mathrm{T} 1 \mathrm{might}$ not be able to read the query messages from R1 and R2 due to interference. This kind of interference is still ineluctable even when $\mathrm{R} 1$ is operating on a different channel with R2. For static readers, the problem can be solved as the two readers keep a certain distance away.
For Mobile RFID readers, however, there would be many chances that several readers are in close proximity such that interference is inevitable. Yet, this problem can be solved when one reader transmits a more powerful signal to a tag than the others. It is reported that, in practice, if signal strength of a certain reader that is communicating with a tag is $13 \mathrm{~dB}$ greater than that of other adjacent readers, no interference will occur [7].

\subsection{Reader-to-Reader Collision}

Figure 1 (b) demonstrates reader-to-reader collision that arises when a neighbor reader R2's strong signal interferes with the weak reflected signal from a tag $\mathrm{T} 1$. Generally, signal strength of a reader is superior to that of a tag and therefore if a frequency channel occupied by $\mathrm{R} 2$ is the same as that of $\mathrm{T} 1$ and $\mathrm{R} 1, \mathrm{R} 1$ is no longer able to listen to T1's response. This problem can be solved when the channel occupied by R1(T1) is different from the channel of R2. In the case of an identical channel being used, it should be assured that R1 and R2 keep their distance long enough such that T1's responding signal strength becomes more powerful than R2's interference signal.

\subsection{Tag Hidden Terminal Problem}

Hidden terminal problem in RFID system was introduced in a paper that proposed PULSE protocol [6]. As depicted in Figure 1 (c), signal from R2 interferes with signal from $\mathrm{R} 1$ to a tag $\mathrm{T} 1$ but the signal from R2 does not reach R1, which is communicating with $\mathrm{T} 1$ and thus $\mathrm{R} 1$ does not recognize the collision that occurs at $\mathrm{T} 1$. The paper [6] also described that this hidden terminal problem can not be solved with simple CSMA technique being used. 


\section{Related Work}

In Colorwave [1], each reader selects any time slot (color) randomly and reads its tag in its own time slot. If a reader selects a timeslot that is the same as that of other neighbor readers and reader collision occurs, the reader will select another new timeslot and send 'kick' messages to those neighbors to let them know the new time slot. Colorwave is not appropriate for Mobile RFID because TDMA scheduling needs reader synchronization and high mobility will generate lots of communication overhead for scheduling. What is worse is that the Colorwave does not utilize multichannel and thus it is necessary that each reader keeps longer distance away than when utilizing multichannel enabled readers in order to avoid reader-toreader collision. This will surely cause to decrease read throughput. Annex G (Dense mode) of Class 1 Generation 2 standard [3] ratified by EPCGlobal describes frequency plan and time-division multiplexing (TDM) method that reader transmission and tag responses are separated spectrally in multichannel environment or temporally in single-channel. The purpose of dense mode is that reader signal does not interfere with tag response although the reader signals still collide with each other and tag signals do. A European evolving standard, Listen Before Talk (LBT) [4] lets a reader first listen on the data channel for any ongoing communication for a specified minimum time and if any signal strength greater than threshold is detected on the channel, then the reader will seek for another empty channel. Dense mode and LBT both solve the reader-to-reader collision problem but still, the multiple reader-to-tag collision problem and the hidden terminal problem are remaining nuisances. USA adopted the Frequency Hopping (FH) method with more than 50 channels whereas LBT [4] is being used in Europe with 15 channels. In Korea, both FH and LBT with 27 channels are adopted. Cole [5] uses a RFID path loss model (1) that can be applicable both in outside and inside buildings to show how far readers should be to avoid reader-to-reader collision. In equation (1), the unit of path loss is presented $\mathrm{dB}$ and symbol $d$ indicates distance from a reader.

$$
\operatorname{PathLoss}(d B)=\left\{\begin{array}{l}
32+25 \log (d), \quad \text { if } 0 \leq d<8 m \\
23+35 \log (d), \quad \text { if } d \geq 8 m
\end{array}\right.
$$

The PULSE protocol [6] separates a control channel from RFID data channels. A reader communicating with tags periodically broadcast beacon messages to the extent greater than its interference range (usually few hundreds meter) through the control channel and readers receiving the beacon messages do not prompt to communicate with tags but rather wait until they do not receive any beacon messages any longer. Through PULSE, any kinds of collision problems can be avoided and also it is feasible for mobile reader environment. Nevertheless, because the neighbor readers in the interference range of the reader are forced not to read the tags, it wastes other channels available. In our proposed scheme, we propose a protocol that circumvents all types of reader collision problems and simultaneously it also enables mobile readers to operate multi-channel concurrently so as to reduce tag reading time. It is based on CSMA and outperforms LBT [4] and PULSE [6].

\section{RFID GENTLE Protocol}

In Section 4.1, we show that the tag hidden terminal problem actually does not exist in practice. Knowing the fact of nonexistence of the tag hidden terminal problem, in section 4.2, we propose Gentle 1 that improves previous approaches [4][6]. In the following section 4.3, we present a mechanism, Gentle 2 that enhances read throughput with sharing tag information among readers. We assume that adjacent channel interference and channel switching time do not exist and type of RFID readers' antenna is omnidirectional.

\subsection{Motivation: Tag Hidden Terminal Problem}

It is insisted in PULSE [6] that the tag hidden terminal problem (fig. 1(c)) can not be overcome with only a simple CSMA methodology. To solve such a problem in PULSE, a reader periodically broadcasts beacon messages further than the scope of interference range on a separate control channel. However, at this point, we strongly doubt whether the tag hidden problem is ever possible in reality. Hence, we verify that tag hidden terminal problem can not exist in real world with capture effect [7] and the RFID path loss model [9].

First, let us calculate the maximum distance that a neighbor reader can interfere with a tag. In [7], it is alleged that, in general, when the signal strength of reader $\mathrm{R} 1$ communicating with a tag is $13 \mathrm{~dB}$ (this value is from real measurement) greater than that of an adjacent reader R2, the adjacent reader R2 does not interfere with the tag, therefore, the tag can read the signal from the reader R1. (This kind of phenomenon is known as capture effect.) Thus, given that a difference between the path loss values of two readers is $13 \mathrm{~dB}$, if we apply it to the equation (1), it can be obtained that, in order to be interference free, the 
distance between the tag and the adjacent reader R2 should be at least approximately 3 times further away than that between the tag and the reader R1. We drive it as following equation where the distances between the reader $\mathrm{R} 1$ and the tag and between the reader $\mathrm{R} 2$ and the tag are denoted $\mathrm{x}$ and $\mathrm{a} * \mathrm{x}$ respectively and transmission power of each reader is signified by $\mathrm{P}$.

$$
\begin{aligned}
& (P-(32+25 \log x))-(P-(32+25 \log a x))>13 \\
& \Rightarrow a>10^{13 / 25} \approx 3.31
\end{aligned}
$$

As an example, given that the distance between a tag and reader R1 is 3 meters (where minimum power that a tag require from a reader for tag operation is $-13 \mathrm{dBm}$ [12], reader transmission power is $30 \mathrm{dBm}$ [8]), the maximum distance that an adjacent reader can interfere with the tag is about 9 meters from the tag.

Next, we investigate the maximum distance that a reader can detect its neighbor's signal. In [8], reader's the maximum transmission power is $30 \mathrm{dBm}^{1}$ and its sensitivity (minimum power of a signal that a reader can detect) is $-101 \mathrm{dBm}$. It means that if the path loss of the signal of a neighbor reader is less than or equal to $131 \mathrm{~dB}(=30+101)$, the reader $\mathrm{R} 1$ can detect its neighbor reader R2's signal. Applying to the equation (1), it can be acquired that a reader is able to detect a signal from any readers that are within 1218 meters.

From the facts above, we can conclude that the maximum distance such that a reader can detect the signal of its neighbor readers is much longer than the maximum distance that a neighbor reader can interfere with a tag. Therefore, it is obviously a wrong argument that a reader cannot detect interference from its neighbor readers located in with a certain distance away. In other words, the tag hidden terminal problem, which PULSE argues, does not exist in CSMA mechanism when we consider the capture effect. As the capture effect value is from real measurement and RFID path loss model [9] represents an average of real values, our conclusion would be approximately close to the reality. The maximum distance which a reader can detect its neighbors' signals could be less than 1218 meters and it does really depend on environment. However, it is sufficient to insist that hidden terminal problem can not actually exist since the maximum distance that a neighbor reader can interfere with a tag is 9 meters, which is 135 times different from 1218 meters. To the best of our knowledge, this paper is the first claim that the tag hidden terminal problem can not exist.

\footnotetext{
${ }^{1}$ The unit $\mathrm{dBm}$ denotes an absolute power level referenced to milliwatt $(\mathrm{mW})$ whereas $\mathrm{dB}$ is a logarithmic unit of measurement that expresses the ratio of two values.
}

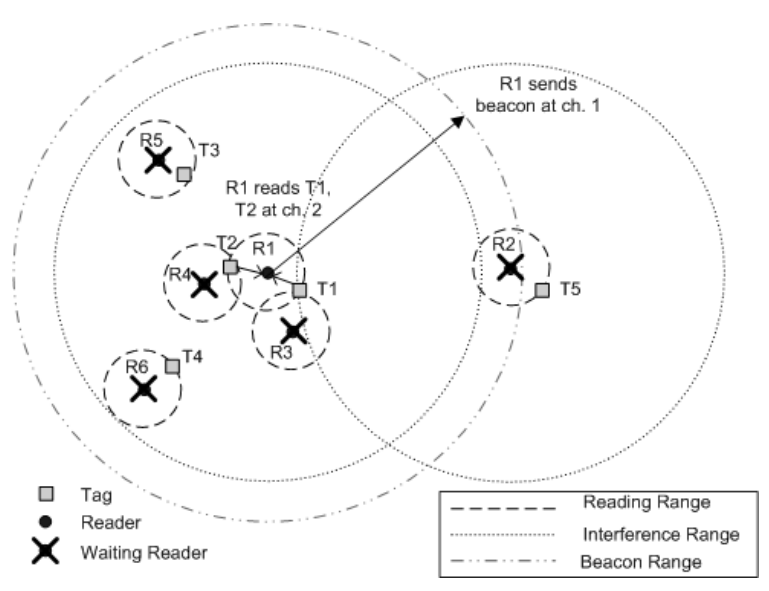

Figure 2. The example of PULSE operation

\subsection{Gentle Protocol 1: Waiting by Beacon}

In the previous section 4.1 , we have claimed that if an adjacent reader interferes with a tag, there is no single instance such that the reader reading tag cannot identify adjacent reader due to long distance between readers. However, it is based on the assumption that the readers are on the same channel and hence if multiple readers are close to one another and the used channels are different, then multiple reader-to-tag collision occurs since the readers cannot detect their neighbors' signals.

Thus, we let one channel be reserved as a control channel that all readers can share with and a reader communicating with a tag periodically sends a beacon message through the control channel. The beacon message is restricted to be sent only within a certain scope, i.e., only up to the distance that multiple readerto-tag collision might occur. The way that a beacon message is sent to other neighbor readers is basically the same but the main difference of our algorithm to the PULSE protocol [6] is that in PULSE, a reader broadcasts a beacon message to much further distance (i.e., about several hundred meters) than our algorithm in order to avoid all types of reader collision including tag hidden terminal problem. Further, PULSE can not benefit from multi-channel whereas Gentle helps increase read throughput using multi-channel. Figure 2 and 3 well illustrate how the PULSE and our proposed algorithm are different.

In figure 2 (PULSE), a reader R1 sends a beacon message periodically far away at ch. 1, i.e., control channel, and many neighbor readers including R2 listen to the message at the shared control channel. The reason why the reader $\mathrm{R} 1$ sends the beacon message up to $\mathrm{R} 2$, which is far away from the $\mathrm{R} 1$, is because $\mathrm{R} 2$ 


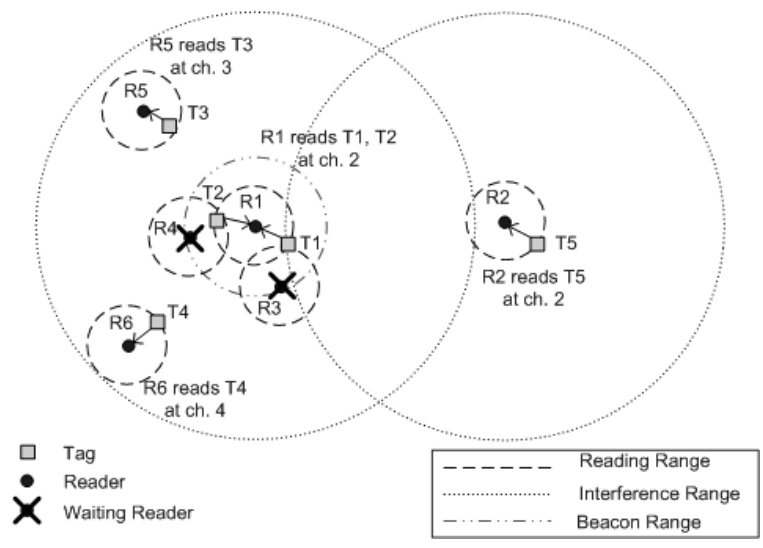

Figure 3. The example of Gentle operation

may affect the tag $\mathrm{T} 1$ communicating with R1 (i.e., tag hidden terminal problem). To avoid the interference, accordingly, the readers R2, R3, R4, R5, and R6 have to wait until they do not receive beacon message from $\mathrm{R} 1$ any more. The reader $\mathrm{R} 1$ reads tag $\mathrm{T} 1$ and $\mathrm{T} 2$ at ch. 2 (i.e., data channel) while neighbor readers wait. In this way, long waiting time for reading tags is required, which will result in reduction of read throughput through the whole network. According to figure 2, for instance, assuming that it takes 1 time unit for a reader to read a tag, the total read throughput is $2 \mathrm{tags} / 2$ time units $=1 \mathrm{tag} /$ time unit (For simplicity, backoff time is not considered in this example). In figure 2 (PULSE), R5 and R6 can read their tags if they use other data channels in order to avoid reader-to-reader collision but they have to wait because they receive beacon message from R2. Therefore, PULSE can not benefit from using multi-(data) channel even if it uses the multi-channel.

On the other hand, according to figure 3 (Gentle), the reader R1 broadcasts a beacon message only up to the reader R3 and R4, which might cause multiple reader-to-tag collision to the tag $\mathrm{T} 1$ and $\mathrm{T} 2$. In the same manner explained in Section 4.1, the tag $\mathrm{T} 1$ can identify R1's signal even in case that both R1 and R2 transmit signals simultaneously since R1's strong signal is superior to very weak one of R2. Thus, only $\mathrm{R} 3$ and $\mathrm{R} 4$ wait while $\mathrm{R} 1$ is reading $\mathrm{T} 1$ and $\mathrm{T} 2$ at channel 2. On the other hand, R5 and R6, which are located in R1's interference range but have not received the beacon message, randomly choose empty data channels (ch. 3 and 4) to avoid reader-to-reader collision and read their own tag, T3 and T4. Since R2 is outside of R1's interference range, there is no readerto-reader collision problem even though R2 uses the channel 2 of R1. In Figure 3, the read throughput is 5tags $/ 2$ time units $=2.5$ tags/time unit according to
Gentle 1 protocol, which is 2.5 times as many as that of PULSE in figure 2.

Using Gentle protocol, therefore, in the case that a reader receives a beacon message from a neighbor, it simply waits for the neighbor's reading. On the other hand, when a reader does not receive any beacon message, it tries to read tags in its reading range through one of available channels. In this way, multiple reader-to-tag collision problem can be solved with sending beacon messages when a reader is closely located in with an interfering reader and when the distance between those readers is long, the readers might use multi-channel that leads to improvement on read throughput. Its entire algorithm including backoff process is shown in figure 4.

\subsection{Gentle Protocol 2: Forwarding}

In static RFID system for inventory management, when more than two readers are involved in reading a tag, it is acceptable that at least one reader reads the tag and forwards the data of tag to the server of RFID system. However, in mobile RFID environment, if more than two readers have the same tag within their range, every reader needs to read the tag. Therefore, we propose a forwarding mechanism (Gentle 2) to increase read throughput of all readers when there is high probability of multiple reader-to-tag collision and the data of tags do not require particular security. The basic idea is that a reader reading tags forwards the data of tags to neighbor readers to want to the same tags on a control channel. The detail mechanism is that the reader reading tags broadcasts the data of a tag by a beacon message whenever it reads each tag. This mechanism makes readers save waiting time for reading tags and decrease the number of attempt to read tags and reduce reader collision that can occur in future. In addition, neighbor readers can get the data of tags before they come close to tags enough. In other words, this mechanism also makes readers expand the range to get the data.

For example, in figure 3, reader R1 forwards tag ID to readers R3 and R4 by beacon message whenever R1 reads a tag. R4 and R3 can get information of tag T1 and $\mathrm{T} 2$ during their waiting time although they do not communicate with the tags T1 and T2. In this way, we can achieve the result that R1, R3, and R4 can read T1 and T2 simultaneously. When we apply the forwarding mechanism to the network topology such as figure 3, the total read throughput is $(2 * 3+3)$ tags $/ 2$ time unit $=$ 4.5 in figure 3. Furthermore, R3 and R4 do not attempt to read $\mathrm{T} 1$ and $\mathrm{T} 2$ after $\mathrm{R} 1$ completes its reading because they already obtained information of the tags from R1. However, if R3 or R4 finds that there exist more tags in its reading range rather than $\mathrm{T} 1$ and $\mathrm{T} 2$, a 


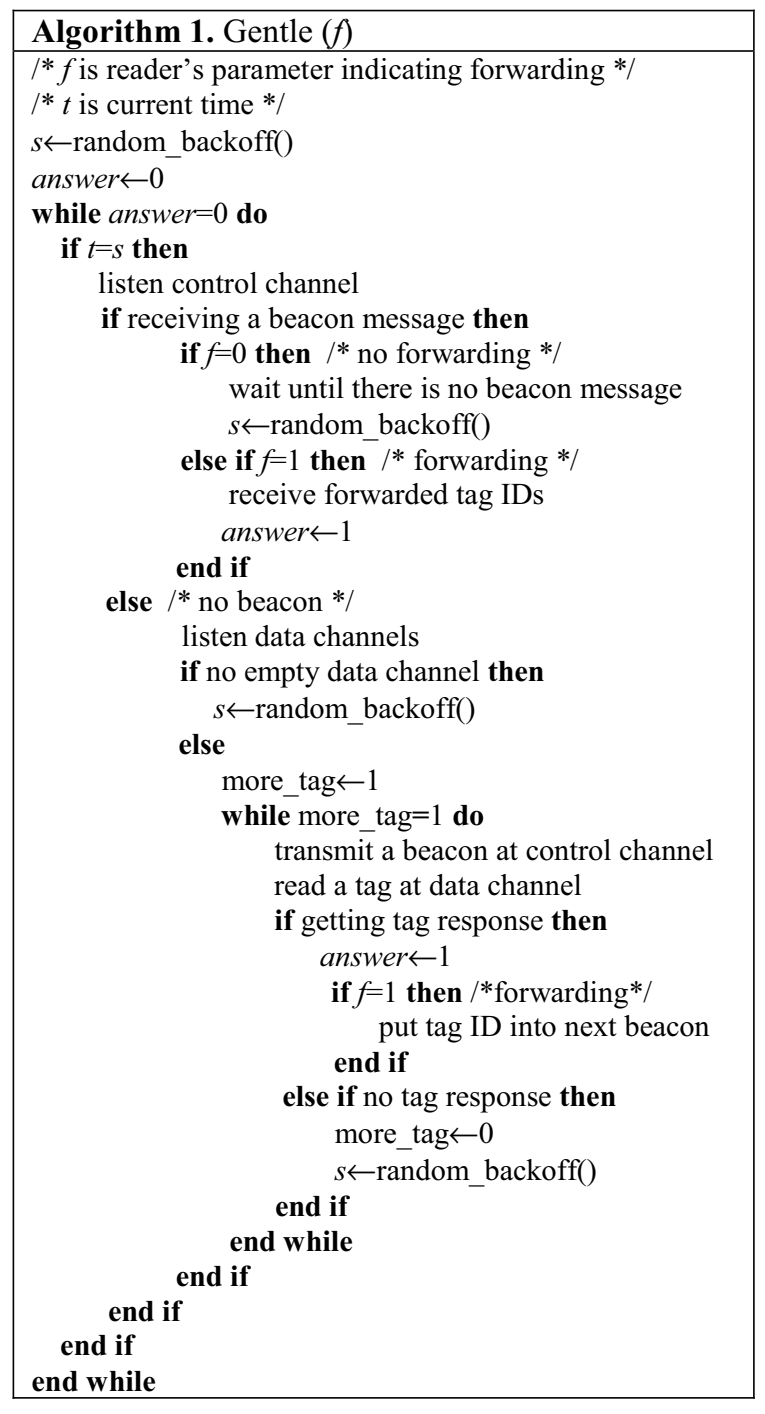

Figure 4. Gentle Pseudo Code

user of R3 or R4 recognizes that there are unread tags and tries to read them directly. In figure 4, we show process of Gentle protocol as pseudo code.

\section{Simulation Results}

To evaluate RFID MAC protocol, we have simulated the Mobile RFID environment with $\mathrm{C}$ language. Table 1 describes the values of parameters for the simulation. We set time to listen channel as $5 \mathrm{~ms}$ based on LBT standard [4] and beacon transmission period as $5 \mathrm{~ms}$ so that a reader can detect a beacon message during listening channel. According to tag anti-collision mechanism (Frame slotted aloha) in standard [13], the slot that a reader reads a tag successfully spends $2.88 \mathrm{~ms}$, the slot that no tag responds is $0.5 \mathrm{~ms}$, and tag collision slot consumes $2.88 \mathrm{~ms}$ when reader data rate is $40 \mathrm{kbps}$. In our simulation, each reader reads about 1 3 tags in its reading range. Thus, we assume that total time spent for reading one tag is about $5 \mathrm{~ms}$ given that a reader uses 16 slots per 1 frame for tag anti-collision. At initial stage of the simulation, we randomly deploy the readers and tags in a room. Each reader moves towards its closest tag in random time. After the reader reads the closest tag using RFID MAC protocol, it attempts to move to the next closest tag, which has been not read by the reader, in another random time and this process repeats. The reason of setting the reader mobility as above is because visitors in museum, as an instance of Mobile RFID application environment, generally get information from the closest articles one by one. A reader does not try to communicate with a tag unless the reader and the tag become close to each other. Each reader stop moving and reading tags after reading all tags in the room. PULSE and Gentle protocols reserve one channel as a control channel (i.e., they have one less number of channels available for tag-reader communication compared to FH and LBT.). In addition, PULSE and Gentle have backoff process choosing one slot in $[0, \ldots, \mathrm{CW}]$ before a reader sends the first beacon message. Gentle sets Maximum Contention Window (CW) to 5 and PULSE sets $\mathrm{CW}$ to 5 or 60 .

Table 1. Parameters for Simulation

\begin{tabular}{|l|l|}
\hline \multicolumn{1}{|c|}{ Parameter } & \multicolumn{1}{c|}{ Value } \\
\hline Simulation range & $15 \times 15\left(\mathrm{~m}^{2}\right)$ \\
\hline Time to read one tag & $5 \mathrm{~ms}$ \\
\hline Time to listen channel & $5 \mathrm{~ms}$ \\
\hline Beacon period & $5 \mathrm{~ms}$ \\
\hline $\begin{array}{l}\text { Maximum contention } \\
\text { window (CW) }\end{array}$ & 5 (PULSE, Gentle), 60 (PULSE) \\
\hline $\begin{array}{l}\text { Multiple reader-to-tag } \\
\text { collision distance }\end{array}$ & $2 \mathrm{~m}$ (default), 3m, 4m, 5m, 6m \\
\hline $\begin{array}{l}\text { Reader-to-reader } \\
\text { collision distance }\end{array}$ & $70 \mathrm{~m}$ \\
\hline No. of readers & $20,40,60$ (default), 80, 100 \\
\hline No. of tags & 60 \\
\hline No. of channels & 10,20 (default), 30, 40, 50 \\
\hline Compared protocols & $\begin{array}{l}\text { FH, LBT, PULSE, Gentle 1, Gentle 2 } \\
\text { (Gentle 1+forwarding) }\end{array}$ \\
\hline
\end{tabular}




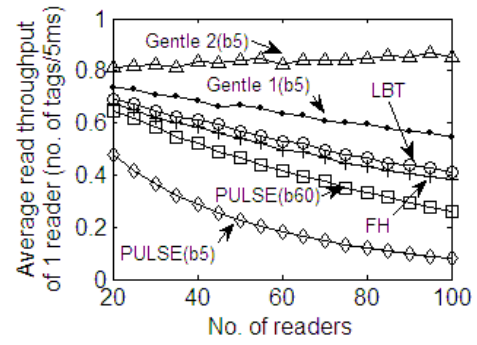

Figure 5. Throughput according to no. of readers

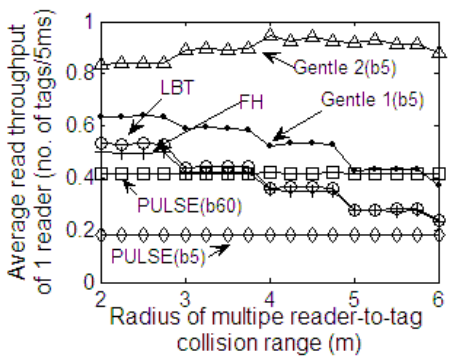

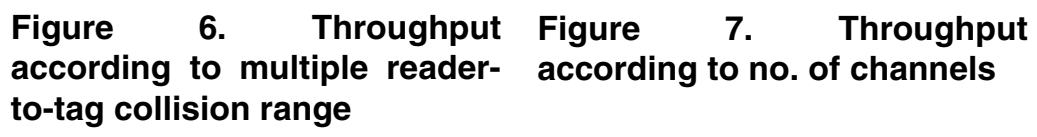

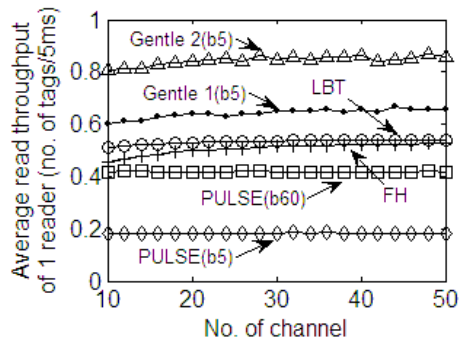

\subsection{Average Read Throughput}

In this section, performance is measured based on average read throughput, i.e., average number of tags that a reader reads per one time unit $(5 \mathrm{~ms})$. Time for deriving average read throughput includes only time for reading tags except moving time and backoff time. Therefore, average read throughput for simulation reflects the probability that a reader reads a tag successfully when it sends query to the tag. For example, if a reader reads each tag successfully every time unit without any collision, the average read throughput of the reader is 1 .

\subsubsection{Comparison using no. of readers}

Figure 5 shows different average read throughput per a reader with variable number of mobile readers. Other parameters are fixed as default values in Table 1. According to the figure 5, read throughput declines as the number of reader increases. Intuitively, it is caused by increasing collision as the number of reader increases. Not like other protocols, however, it is observed that read throughput does not decrease for Gentle 2 due to the forwarding mechanism. LBT shows little better performance overall than FH by reducing the reader-to-reader collision. PULSE has low average read throughput due to many reader-to-reader collision and multiple reader-to-tag collisions.

\subsubsection{Comparison using range of multiple reader- to-tag collision}

Figure 6 depicts the result from simulation with varying distances between readers that might bring in multiple reader-to-tag collision. Other parameters including reader-to-reader collision distance are fixed as default values in Table 1. A stair graph is represented since radius of a reader's reading range is set to 1 . In general, read throughput decreases as the scope of multiple reader-to-tag collision increases. However, since the extent of multiple reader-to-tag collision and beacon transmission are the same, Gentle 2 performs better by forwarding as the range increases. It is also shown that Gentle 1 is the second most superior to other protocols and no difference on the performance of LBT and FH is found as multiple reader-to-tag collision gets large. Because LBT cannot solve multiple reader-to-tag collision, although LBT prevents reader-to-reader collision, the performance of LBT becomes undifferentiated from that of $\mathrm{FH}$ as the possibility for occurrence of multiple reader-to-tag collision increases. PULSE has no difference of performance as multiple reader-to-tag collision range increases. This is because PULSE has so many readerto-reader collisions compared to multiple reader-to-tag collisions and most multiple reader-to-tag collisions occurs with reader-to-reader collisions in PULSE.

\subsubsection{Comparison using no. of channel}

Figure 7 shows average read throughput changes when using different protocols with variable number of channels. Other parameters are fixed as default values in Table 1. As the number of channel increases, there is slight improvement on read throughput for all the protocols experimented except PULSE protocol, which is due to multi-channel unavailability. It is also demonstrated that the performance of LBT with 10 channels is analogous to that of FH with 50 channels, which ascertains that it is fundamentally more essential to invent an efficient reader anti-collision algorithm rather than increasing the number of channels for improving read throughput.

\section{Conclusion and future work}

This paper handles reader collision problem that prevents RFID readers from reading tags and we proposes Gentle protocol that outperforms the existing 
reader anti-collision protocols. The basic idea is that readers avoid multiple reader-to-tag collision by using beacon messages when they are close one another and reader-to-reader collision by using multi-channel when the distance between those readers is long. Gentle protocol can avoid two kinds of reader collision efficiently and take advantage of given RFID multichannel resource as well as increase read throughput. We also find that hidden terminal problem [6] does not exist. In addition, if the reader reading tags broadcasts the data of a tag by a beacon message, it forwards the data of the tag to neighbor readers and then read throughput of all readers increase and reading range of readers is expanded. When using forwarding mechanism, read throughput increase with high possibility of multiple reader-to-tag collision. Gentle protocol would be proper solution to multi-channel Mobile RFID environment where readers have high mobility and need a distributed reader anti-collision protocol.

\section{References}

[1] J. Waldrop, D. Engels, and S. Sarma, "Colorwave: An Anticollision Algorithm for the Reader Collision Problem," in Proc. IEEE ICC'03, May 2003.

[2] Z. Zhou, H. Gupta, S. Das, and X. Zhu, "Slotted Scheduled Tag Access in Multi-Reader RFID Systems," in Proc. IEEE ICNP, October 2007.

[3] "EPCTM Radio-Frequency Identification Protocols Class-1 Generation-2 UHF RFID Protocol for Communications at $860 \mathrm{MHz}-960 \mathrm{MHz}$ Version 1.0.8," EPCglobal, 2004.

[4] "ETSI EN 302 208-1 v1.2.1," ETSI, 2008.

[5] K. Leong, M. Ng, and P. Cole, "The Reader Collision Problem in RFID Systems," in Proc. IEEE MAPE, August 2005.

[6] S. Birari and S. Iyer, "PULSE: A MAC Protocol for RFID Networks," in Proc. the $1^{\text {st }}$ International Workshop on RFID and Ubiquitous Sensor Networks (USN'05), December 2005.

[7] Chris Turner, "White paper on the dense reader problem in Europe," ISO-IEC JTC1/SC31/W4/SG3 Ad hoc committee on dense reader problem, 2006.

[8] "Passive, Battery-assisted Passive and Active Tags: A Technical Comparison," White paper, Intelleflex Confidential.

[9] "Standard on Radio Specification for Mobile RFID Reader," TTA, TTAS.KO-06.0119, 2006. Available: http://ww.tta.or.kr

[10] J. Myung and W. Lee, "Adaptive Splitting Protocols for RFID Tag Collision Arbitration," in Proc. ACM MobiHoc, May 2006.

[11] J. Kim, E. Kim, D. Kim, W. Lee, and K. Suh, "Optimized Transmission Power Control of Interrogators for Collision Arbitration in UHF RFID Systems," IEEE Communications Letters, Vol. 11, No. 1, pp. 22-24, January 2007.
[12] U. Karthaus and M. Fischer, "Fully Integrated Passive UHF RFID Transponder IC With $16.7-\mu \mathrm{W}$ Minimum RF Input Power," IEEE Journal of Solid-State Circuit, Vol. 38, No. 10, October 2003.

[13] "Information technology - Radio frequency identification for item management - Part 6: Parameters for air interface communications at $860 \mathrm{MHz}$ to $960 \mathrm{MHz}$," ISO/IEC 18000-6, 2004.

[14] F. Aurenhammer, "Voronoi Diagrams - A Survey of a Fundamental Geometric Data Structure," ACM Computing Surveys, Vol. 23, No. 3, pp. 345-405, September 1991.

[15] C. Seidler, "RFID Opportunities for Mobile Telecommunication Services," ITU-T Lighthouse Technical Paper, 2005. Availabe: http://www.itu.int/ITUT/techwatch/rfid.pdf 